\title{
Twisted mass lattice QCD: Recent developments and results
}

\author{
Andrea Shindler* \\ NIC, DESY Platanenallee 6 \\ 15738 Zeuthen, Germany \\ E-mail: andrea.shindleredesy.de
}

I review recent theoretical developments and numerical results of twisted mass QCD. I argue that, combined with an efficient algorithm, twisted mass QCD can be an attractive QCD lattice action, to perform large scale simulations at small pion masses, where a matching with chiral perturbation theory can be performed. Open issues like flavour breaking effects are also addressed.

DESY 05-216

SFB /CPP-05-73

XXIIIrd International Symposium on Lattice Field Theory

25-30 July 2005

Trinity College, Dublin, Ireland

*Speaker. 


\section{Cutoff effects and renormalization}

Phenomenological results from simulations of lattice QCD to compare with experiments should be obtained with all the systematic uncertainties under control. The first requirement is to have an efficient algorithm to simulate $N_{\mathrm{f}}=2$ dynamical light quarks with the possibility to include $1(+1)$ heavier quarks. The algorithm should allow, in a reasonable time, to reach small pion masses $\left(m_{\pi}<300 \mathrm{MeV}\right)$ where a matching with chiral perturbation theory $(\chi \mathrm{PT})$ should become possible, and to simulate a large enough volume $(L \geq 2 \mathrm{fm})$. The second requirement is to have a lattice action with good scaling and simplified renormalization properties, as close as possible to the renormalization of continuum QCD. The topic I want to address in this contribution is if lattice twisted mass QCD (tmQCD), combined with a suitable algorithm, is a possible lattice action that fulfills these requirements.

\subsection{Lattice QCD action}

Despite the only rather recent interest, the tmQCD fermionic lattice action has a long history. It was introduced in [1] as a tool to study spontaneous parity and flavour symmetry breaking. In [2] it was proved that lattice tmQCD is an alternative discretization of lattice QCD. The lattice QCD action

$$
S=S_{g}[U]+S_{F}[U, \psi, \bar{\psi}]
$$

has a fermionic part given by tmQCD

$$
S_{F}=a^{4} \sum_{x}\left\{\bar{\psi}(x)\left[D[U]+m_{0}+i \mu \gamma_{5} \tau^{3}\right] \psi(x)\right\}
$$

and for the moment we leave unspecified the gauge part $S_{g}$. In eq. (1.2) $D[U]$ is the massless Wilson-Dirac operator

$$
D[U]=\frac{1}{2}\left[\gamma_{\mu}\left(\nabla_{\mu}+\nabla_{\mu}^{*}\right)-a \nabla_{\mu}^{*} \nabla_{\mu}\right]
$$

$m_{0}$ is the untwisted bare quark mass parameter, $\mu$ is the bare twisted quark mass, and $\tau^{3}$ is the third Pauli matrix acting in flavour space. In [3] it was shown that the standard framework of the Symanzik improvement program, works in the similar way as for usual Wilson fermions. In particular for spectral quantities no further improvement coefficients are needed. A set of scaling tests have been performed, using the non-perturbatively improved clover action with twisted mass, in small [4] and large [5] volume, confirming that the usual Symanzik improvement program can be applied also for tmQCD.

In a remarkable paper of Frezzotti and Rossi [6] a step forward was made. It was proved that parity even correlators of multiplicatively renormalizable fields, are free from $\mathrm{O}(a)$ effects, and so no improvement coefficients are needed (automatic $\mathrm{O}(a)$ improvement), if the target continuum theory is fully twisted ${ }^{1}$. The proof in [6] is based on a set of spurionic symmetries of the lattice action. Here we give a simpler proof based on the symmetries of the continuum QCD action (see appendix A of [7] for an analogous proof). The Symanzik [8, 9, 10, 11, 12] effective action reads

$$
S_{\text {eff }}=S_{0}+a S_{1}+\ldots
$$

\footnotetext{
${ }^{1}$ To obtain automatic $\mathrm{O}(a)$ improvement in [6] also other possibilities were exploited. Here we will concentrate on the automatic $\mathrm{O}(a)$ improvement that is used in numerical simulations.
} 
and we are interested in a continuum target theory where the physical quark mass is fully given by the renormalized twisted mass $\mu_{\mathrm{R}}$ (fully twisted theory)

$$
S_{0}=\int d^{4} x \bar{\psi}(x)\left[\gamma_{\mu} D_{\mu}+\mathrm{i} \mu_{\mathrm{R}} \gamma_{5} \tau^{3}\right] \psi(x)
$$

The correction terms in the effective action are given by

$$
S_{1}=\int d^{4} y \mathscr{L}_{1}(y) \quad \mathscr{L}_{1}(y)=\sum_{i} c_{i} \mathscr{O}_{i}(y)
$$

where the dimension five operators classified on the basis of the symmetries of the lattice action are given by [3]

$$
\mathscr{O}_{1}=\bar{\psi} \sigma_{\mu \nu} F_{\mu \nu} \psi \quad \mathscr{O}_{2}=\mu^{2} \bar{\psi} \psi \quad \mathscr{O}_{3}=\Lambda^{2} \bar{\psi} \psi
$$

where $\Lambda$ is an energy scale of the order of the QCD scale $\Lambda_{\mathrm{QCD}}$. The operator $\mathscr{O}_{1}$ is the usual clover term. The operators $\mathscr{O}_{2}$ and $\mathscr{O}_{3}$ are related to the renormalization of the untwisted quark mass. Since we are interested in a continuum target theory where the untwisted quark mass vanishes, the operator $\mathscr{O}_{3}$ parameterizes the mass independent $\mathrm{O}(a)$ uncertainties in the critical mass. We consider now a general multiplicatively renormalizable multilocal field that in the effective theory is represented by the effective field

$$
\Phi_{\mathrm{eff}}=\Phi_{0}+a \Phi_{1}+\ldots
$$

A lattice correlation function of the field $\Phi$ to order $a$ is given by

$$
\langle\Phi\rangle=\left\langle\Phi_{0}\right\rangle_{0}+a \int d^{4} y\left\langle\Phi_{0} \mathscr{L}_{1}(y)\right\rangle_{0}+a\left\langle\Phi_{1}\right\rangle_{0}+\ldots
$$

where the expectation values on the r.h.s are to be taken in the continuum theory with action $S_{0}$. The key point is that the continuum action (1.5) is symmetric under the following parity transformation

$$
\begin{aligned}
& \psi(x) \longrightarrow \gamma_{0}\left(i \gamma_{5} \tau^{3}\right) \psi\left(x_{0},-\mathbf{x}\right) \\
& \bar{\psi}(x) \longrightarrow \bar{\psi}\left(x_{0},-\mathbf{x}\right)\left(i \gamma_{5} \tau^{3}\right) \gamma_{0}
\end{aligned}
$$

and that all the operators in eq. (1.7), of the Symanzik expansion of the lattice action, are odd under the parity symmetry of the continuum action. If the operator $\Phi_{0}$ is parity even, the second term in the r.h.s. of eq. (1.9) vanishes, and $\Phi_{1}$, being of one dimension higher, is parity odd: for the same reason the third term in the r.h.s of eq. (1.9) vanishes. Possible contact terms coming from the second term amount to a redefinition of $\Phi_{1}$ and so do not harm the proof.

It is then also clear that in order to achieve automatic $\mathrm{O}(a)$ improvement, the continuum target theory must have a vanishing untwisted quark mass $m_{\mathrm{R}}$, otherwise the standard mass term $m_{\mathrm{R}} \bar{\psi} \psi$ will break the parity symmetry of the continuum action defined before. The most natural way to achieve this on the lattice is by setting the untwisted bare quark mass to its critical value $m_{0}=m_{\mathrm{c}}$. The proof also shows that a possible uncertainty of $\mathrm{O}(a)$ in the critical mass does not wash out automatic $\mathrm{O}(a)$ improvement since these uncertainties, are odd under parity. A remark is in order now. We take the polar mass defined in [3]

$$
M=\sqrt{\mu^{2}+m_{\mathrm{q}}^{2}}=\sqrt{\mu^{2}+\left(\eta_{1} a \Lambda^{2}\right)^{2}} ; \quad m_{\mathrm{q}}=m_{0}-m_{\mathrm{c}}
$$


where the $\eta_{1}$ term parameterizes the mass independent $\mathrm{O}(a)$ uncertainties in the value of the untwisted quark mass $m_{\mathrm{q}}$. Expanding in powers of $a$ we have

$$
M \simeq \mu\left[1+\frac{\eta_{1} a^{2} \Lambda^{4}}{2 \mu^{2}}+O\left(a^{4}\right)\right]
$$

We observe immediately that as soon $\mu<a \Lambda^{2}$, even if parametrically $\mathrm{O}(a)$ terms are absent in (1.13), there is a term of $\mathrm{O}\left(a^{2}\right)$ with a coefficient that tends to diverge as soon $\mu$ is made smaller and smaller. From this example we can conclude that to have an effective automatic $\mathrm{O}(a)$ improvement, without big $\mathrm{O}\left(a^{2}\right)$ effects, with a generic choice of the critical mass, such that the uncertainties in the untwisted quark mass are of order $a \Lambda^{2}$, we need to have the constraint $\mu>a \Lambda^{2}$. It has been shown in [0] that these cutoff effects that diverges at small quark masses, so called infrared divergent (IR) cutoff effects, are a general property of tmQCD. These dangerous cutoff effects are removed by an appropriate choice of the critical mass.

\section{$1.2 \mathrm{O}(a)$ improvement and small pion masses}

The $\mathrm{O}(a)$ uncertainties of the untwisted quark mass depend on how the critical line is fixed, hence the choice of the critical mass has to be discussed with care. The issue was raised by the work of Aoki and Bär [13] and by the numerical results obtained in [14]. This problem has been further analyzed in several aspects [15, 7, 16]. In [13, 15, 16, the theoretical framework is twisted mass chiral perturbation theory $(\operatorname{tm} \chi \mathrm{PT})$ [17] where the cutoff effects are included in the chiral lagrangian along the lines of [18, 19]. In this framework a power counting scheme that includes quark mass and lattice spacing has to be specified. In particular in [13] the power counting was $\mu \sim a^{2} \Lambda^{3}$ while in [15] it was $\mu \sim a \Lambda^{2}$. We stress here that this approach for the description of lattice data, does not require a continuum extrapolation, hence the power counting scheme does not mean that $\mu$ goes to zero in the continuum limit but represents only an order of magnitude equality. Both these works [13, 15] agree on the fact that choosing the critical mass imposing a vanishing PCAC quark mass

$$
m_{\mathrm{PCAC}}=\frac{\sum_{\mathbf{x}}\left\langle\partial_{0} A_{0}^{a}(x) P^{a}(0)\right\rangle}{2 \sum_{\mathbf{x}}\left\langle P^{a}(x) P^{a}(0)\right\rangle} \quad a=1,2
$$

where

$$
\begin{aligned}
A_{\mu}^{a}(x) & =\bar{\psi}(x) \gamma_{\mu} \gamma_{5} \frac{\tau^{a}}{2} \psi(x) \\
P^{a}(x) & =\bar{\psi}(x) \gamma_{5} \frac{\tau^{a}}{2} \psi(x)
\end{aligned}
$$

allows to have automatic $\mathrm{O}(a)$ improvement, and in particular down to quark masses that fulfill $\mu \simeq a^{2} \Lambda^{3}$ for [13] and $a^{2} \Lambda^{3}<\mu<a \Lambda^{2}$ for [15 $]^{2}$. In [7] a Symanzik expansion along the lines of [12] was performed confirming the results of [13, 15].

A possible practical procedure is then to compute for a fixed value of $\mu$ the critical mass $m_{\mathrm{c}}$ from the vanishing PCAC mass, and then to extrapolate the set of critical masses obtained for different values of $\mu$ to $\mu=0$ (method A). This procedure has been used in [20, 21]. In fig. 1 a typical extrapolation of the critical mass to $\mu=0$ is shown. With this procedure the $\mathrm{O}(a)$

\footnotetext{
${ }^{2}$ We will see in section 3.2 that the phase structure of $N_{\mathrm{f}}=2$ dynamical Wilson fermions does not allow anyway the twisted mass to be smaller then $\mu_{\mathrm{c}} \sim a^{2} \Lambda^{3}$.
} 
uncertainties of the critical mass are fixed in such a way that, for a generic value of $\mu$, the dangerous $a \Lambda^{2}$ cutoff effects in the untwisted quark mass are absent. The slope of the curve is proportional

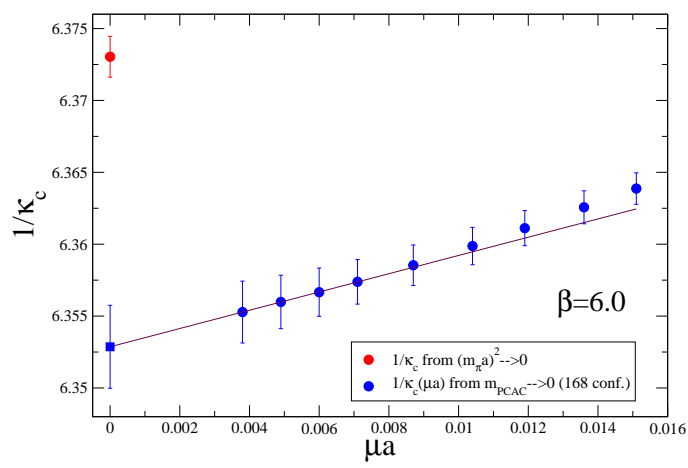

Figure 1: Determination of the critical mass $m_{\mathrm{c}}\left(\kappa_{\mathrm{c}}^{-1}=2 a m_{\mathrm{c}}+8\right)$ for given values of $\mu$ at $\beta=6.0$, and extrapolation to $\mu=0$. The red point is the critical mass determined with method $\mathbf{C}$ (see text). The difference between the two determination of the critical mass should be an $\mathrm{O}(a)$.

to $\mathrm{O}(a)$ cutoff effects related to the discretization errors of the PCAC mass [7, 22, 16. We remind that it is not surprising that the PCAC mass is not automatically $\mathrm{O}(a)$ improved since it is an odd quantity under the parity transformation of eqs. (1.10, 1.11).

In [23] the extrapolation to $\mu=0$ is not performed and each value of the critical mass has been used for the corresponding value of $\mu$ used in the simulations (method $\mathbf{B}$ ). With this method the $\mathrm{O}(a)$ cutoff effects of the critical mass are obviously fixed in such a way that the untwisted quark mass is always vanishing for all the simulation points.

These two methods, even if they give different cutoff effects to the critical mass, are perfectly good in order to achieve automatic $\mathrm{O}(a)$ improvement. Another possible way to fix the critical mass, expecially practical for expensive dynamical simulations, is to compute the critical mass, using the PCAC relation at the smallest value of $\mu$, and then use this critical mass for all the simulation points at heavier masses.

Using methods $\mathbf{A}$ and $\mathbf{B}$ a set of quenched studies [24, 14, 25, 23, 20, 21, 26, have been performed to check the result of [6] and to gain experience with this formulation of lattice QCD. An interesting quantity to compute with tmQCD is the pseudoscalar decay constant $f_{\mathrm{PS}}$. As it was noted in [5, 28, 24], the computation of $f_{\mathrm{PS}}$ does not require any renormalization constant, in contrast of ordinary Wilson fermions, and moreover given automatic $\mathrm{O}(a)$ improvement, does not need the computation of any improvement coefficient. Thus the situation for this quantity is like with overlap fermions. In fig. 2 (left panel) the continuum limit of $r_{0} f_{\mathrm{PS}}{ }^{3}$, the critical mass being computed with method $\mathbf{A}$, is shown as a function of $\left(a / r_{0}\right)^{2}$. The scaling is consistent with being of $\mathrm{O}\left(a^{2}\right)$, and moreover the $\mathrm{O}\left(a^{2}\right)$ effects are rather small for all the pseudoscalar masses investigated down to $m_{\mathrm{PS}}=272 \mathrm{MeV}$. The right panel of fig. 2 shows the chiral behaviour of the continuum pseudoscalar decay constant, compared with the non-perturbatively $\mathrm{O}(a)$ improved data of [27].

\footnotetext{
${ }^{3}$ The values of $r_{0} / a, r_{0}=0.5 \mathrm{fm}$ being the Sommer scale [29], are taken from [30].
} 

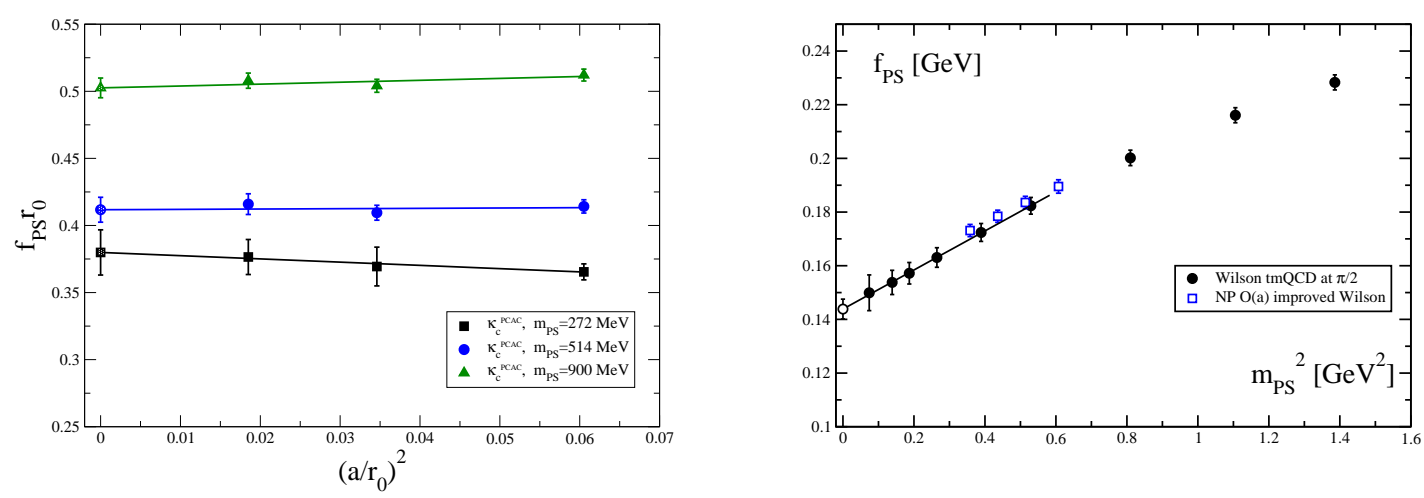

Figure 2: Left panel: scaling behaviour of $r_{0} f_{\mathrm{PS}}$ for 3 fixed values of $r_{0} m_{\mathrm{PS}}$. Right panel: Continuum limit values for $f_{\mathrm{PS}}$ as a function of $m_{\mathrm{PS}}^{2}$ in physical units. The empty squares are taken from [27].

We remark that this comparison is purely illustrative since it is in the quenched approximation, and the simulations with clover fermions had to stop around $m_{\mathrm{PS}} \simeq 500 \mathrm{MeV}$ due to the appearance of exceptional configuration. To see the potential of tmQCD another interesting phenomenological

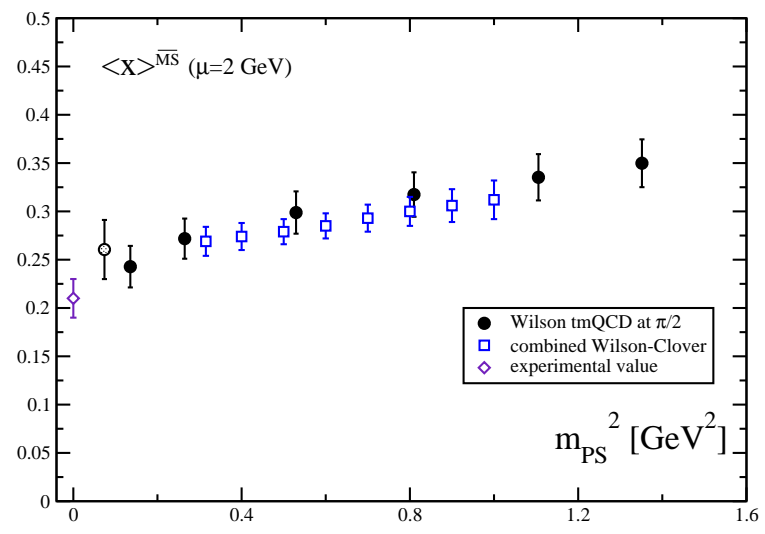

Figure 3: $\langle x\rangle^{\overline{\mathrm{MS}}}(\mu=2 \mathrm{GeV})$ extrapolated to the continuum as a function of the pion mass. Open squares represent results that are obtained from a combined continuum extrapolation of earlier Wilson and cloverWilson simulations [31]. The filled circles represent results using Wilson twisted mass fermions [32]. The open circle denotes a result which is not corrected for finite size effects and the diamond corresponds to the experimental point.

quantity is the average momentum carried by valence quarks in a pion $(\langle x\rangle)$. In [32] results using tmQCD were presented. Here we concentrate on the chiral behaviour in the continuum, having in mind that the renormalization has been performed already in a non-perturbative way [33, 31]. Fig. 3 shows that in principle also for this quantity small pseudoscalar masses $m_{\mathrm{PS}}<300 \mathrm{MeV}$ can be reached, opening the possibility of a safe chiral extrapolation. 

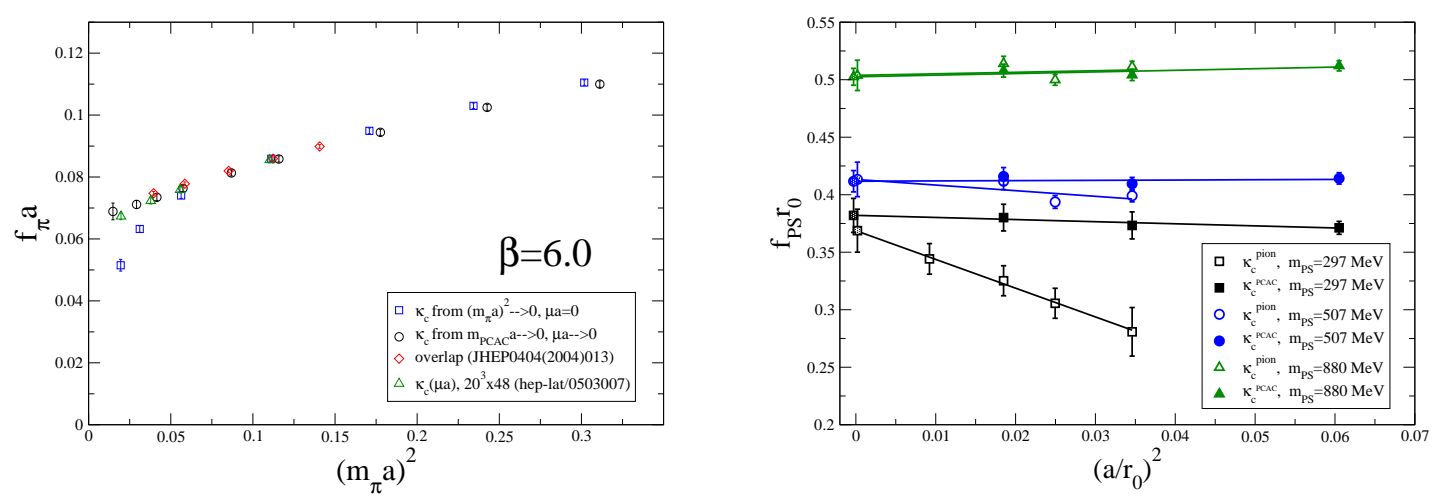

Figure 4: Left panel: comparison of the chiral behaviour at fixed lattice spacing $(\beta=6.0)$ of the pseudoscalar decay constant computed using method $\mathbf{A}, \mathbf{B}, \mathbf{C}$ and with results obtained with overlap fermions. Right panel: unconstrained continuum limit, for several values of fixed charge pion masses, of $r_{0} f_{\mathrm{PS}}$ performed using method $\mathbf{A}$ and $\mathbf{C}$ to determine the critical mass.

In [14] to obtain automatic $\mathrm{O}(a)$ improvement the critical mass $m_{\mathrm{c}}$ was computed extrapolating the squared pseudoscalar mass to the chiral limit using data from the pure Wilson theory (method C). Using this determination of $m_{\mathrm{c}}$ several quantities were computed. In particular in the left panel of fig. $⿴$ there is a comparison of the chiral behaviour at fixed lattice spacing $(\beta=6.0)$ of the pseudoscalar decay constant computed using method $\mathbf{A}, \mathbf{B}, \mathbf{C}$ and with results obtained with overlap fermions [34]. While methods $\mathbf{A}, \mathbf{B}$ and the overlap data are all consistent within the statistical errors, ${ }^{4}$ the data obtained using method $\mathbf{C}$ to fix the critical mass, show a "bending" towards the chiral limit. The same phenomenon was observed also for the vector mass [14]. The "bending" phenomenon appeared exactly when $\mu \simeq a \Lambda^{2}$. Having in mind the caveat observed before in the proof of automatic $\mathrm{O}(a)$ improvement, this indicates that the extraction of the critical mass with method $\mathbf{C}$ leaves the dangerous $a \Lambda^{2}$ in the untwisted quark mass uncanceled. This is numerically confirmed by the results of [21], showed in the right panel of fig. 4 , since using method $\mathbf{A}$ and $\mathbf{C}$ to determine the critical mass, a consistent continuum limit is obtained, showing also that method $\mathbf{C}$ induces big $\mathrm{O}\left(a^{2}\right)$ effects and a reduced scaling window.

A description of the "bending" phenomenon at fixed lattice spacing, has been obtained in [22] using $\chi \mathrm{PT}$, as it is shown in the left panel of fig. 5, where a fit to available quenched data is performed on the ratio $R=\frac{a^{2} m_{\mathrm{PS}}^{2}}{a \mu}$. This analysis shows also that $\chi \mathrm{PT}$ theory is able to describe the lattice data up to $\mu \simeq 80 \mathrm{MeV}$. It is reassuring that using method $\mathbf{A}$ to determine the critical mass and restricting the data to the region were $\chi \mathrm{PT}$ is applicable the ratio $R$ is flat (right panel of fig. 5) consistently with continuum $\chi \mathrm{PT}$ (up to chiral logs). In [7], based on the observation that the big $\mathrm{O}\left(a^{2}\right)$ effects come from uncanceled $\mathrm{O}(a)$ of the PCAC mass, to eliminate the "bending" phenomenon has been proposed to use a non-perturbatively improved tmQCD action. This approach has been numerically tested in [35], and as it can be seen in fig. 6 indeed it confirms that the "bending" phenomenon also in this case it is not present.

\footnotetext{
${ }^{4} \mathrm{We}$ recall here that since the comparison is made at fi xed lattice spacing the data in principle could disagree due to different cutoff effects.
} 

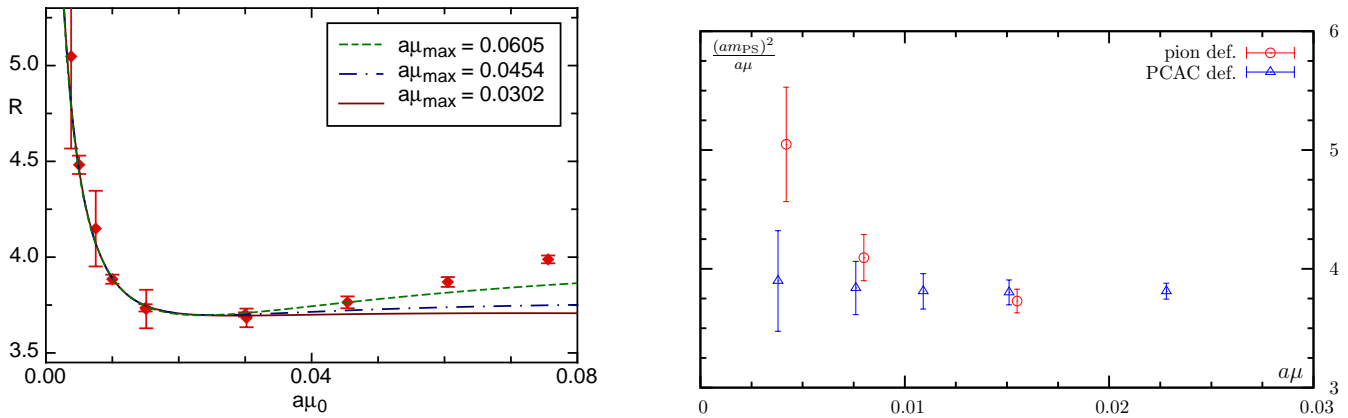

Figure 5: Left panel: bending phenomenon at $\beta=6.0$ on the ratio $R=\frac{a^{2} m_{\mathrm{PS}}^{2}}{a \mu}$ and its description with $\chi \mathrm{PT}$. Right panel: comparison of the ration $R$ using method $\mathbf{A}$ and $\mathbf{C}$ to determine the critical mass.
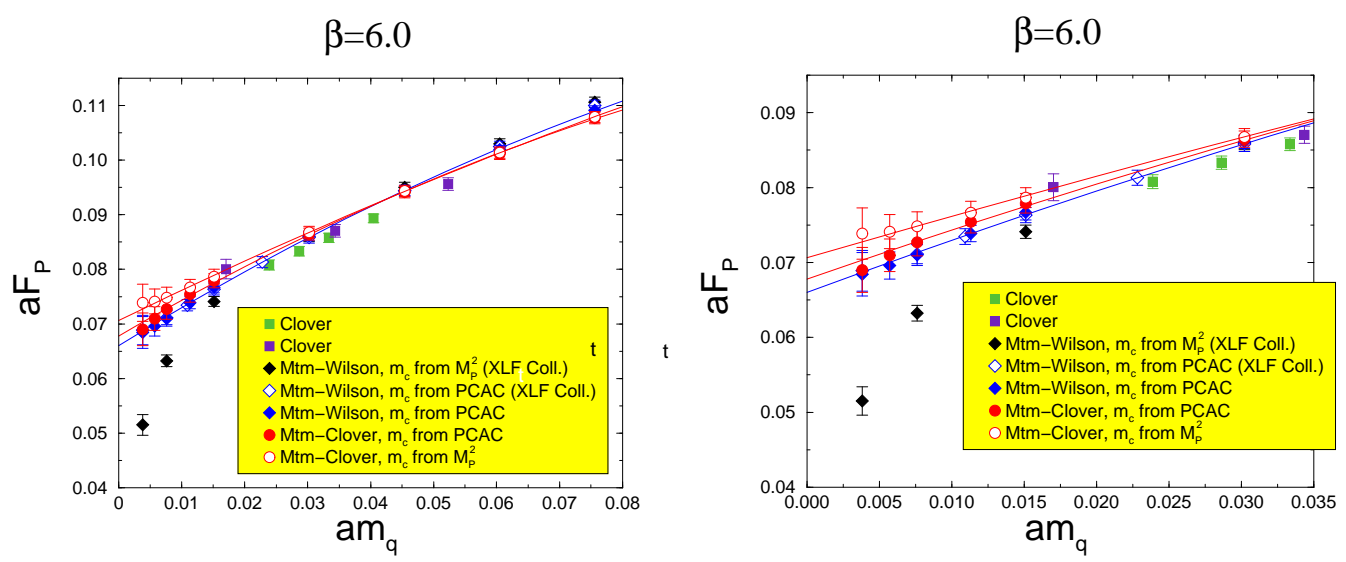

Figure 6: Comparison of the chiral behaviour at fixed lattice spacing ( $\beta=6.0$ ) of the pseudoscalar decay constant computed using method $\mathbf{A}$ and $\mathbf{C}$ for both tmQCD and non-perturbatively improved tmQCD.

\subsection{Renormalization}

In [36] it has been given a construction of a Schrödinger functional (SF) with twisted boundary conditions that preserves the nice properties of $\mathrm{O}(a)$ improvement without bulk improvement coefficients; (see [37] for a possible alternative to this construction). The construction is based on the consideration that in a finite volume with suitable boundary condition the Wilson theory in the chiral limit is $\mathrm{O}(a)$ improved, and it makes use of orbifolding techniques (see [38] for an application of orbifolding techniques to Ginsparg-Wilson fermions).

A simple way to visualize the construction is to repeat the proof of automatic $\mathrm{O}(a)$ improvement given in section 1.1, where now since we are in a finite volume with suitable boundary conditions, the twisted mass could be safely sent to zero. Then the new boundary projectors [36] $Q_{ \pm}=\frac{1}{2}\left(1+\mathrm{i} \gamma_{0} \gamma_{5} \tau^{3}\right)$ commute with the previous parity transformation (as the twisted mass term in infinite volume). It is very important to note that the new boundary projectors can be obtained performing a chiral rotation of the original projectors in the standard SF framework [39]. An important consequence is that the running of the coupling constant, should be identical to the running computed with the "old" SF [40]. The $\mathrm{O}(a)$ uncertainties in the critical mass do not harm the $\mathrm{O}(a)$ 
improvement.

\section{Flavour symmetry}

When tmQCD is used to define the standard QCD correlation functions some of the physical symmetries are restored only in the continuum limit. In particular flavour and parity symmetries. The explicit breaking of flavour symmetry generates for example splitting between charged and neutral pions, while the absence of parity symmetry, gives as a consequence the appearance of states of opposite parity in the spectral decomposition of usual correlators. Both these phenomena are expected to vanish, at maximal twist, with a rate of $\mathrm{O}\left(a^{2}\right)$ [41]. Here we concentrate on the flavour symmetry breaking.

To fix the notation we recall some basic definitions. The charged pseudoscalar currents are given by

$$
P^{ \pm}(x)=\bar{\psi}(x) \gamma_{5} \frac{\tau^{ \pm}}{2} \psi(x) \quad \tau^{ \pm}=\frac{\tau^{1} \pm \mathrm{i} \tau^{2}}{2}
$$

and a possible interpolating field for the neutral pion is the scalar current

$$
S^{0}(x)=\bar{\psi}(x) \psi(x)
$$

The charged and neutral pseudoscalar masses can be extracted by the following correlators

$$
\begin{aligned}
& C_{\pi^{+}}\left(x_{0}\right)=a^{3} \sum_{\mathbf{x}}\left\langle P^{+}(x) P^{-}(0)\right\rangle \quad C_{\pi^{0}}\left(x_{0}\right)=a^{3} \sum_{\mathbf{x}}\left\langle S^{0}(x) S^{0}(0)\right\rangle \\
& C_{\pi^{0}}\left(x_{0}\right)=a^{3} \sum_{\mathbf{x}}\{\langle-\operatorname{tr}[G(0, x) G(x, 0)]+\operatorname{tr}[G(x, x)] \operatorname{tr}[G(0,0)]\rangle\}
\end{aligned}
$$

where $G(x, y)$ is the fermionic propagator. In [42] a pilot quenched study has been preformed to study flavour breaking effects with tmQCD. For the neutral pseudoscalar correlator in eq. (2.4) a first possibility is to study only the connected part. In the quenched approximation it is still possible to interprete the connected part in terms of local operators. The reason is that one could think the connected part as coming from Wick contractions obtained using the Osterwalder-Seiler (OS) [43] action

$$
S_{\mathrm{OS}}=a^{4} \sum_{x}\left\{\bar{\psi}(x)\left[D[U]+m_{0}+i \mu \gamma_{5}\right] \psi(x)\right\} .
$$

This action has a trivial flavour structure and so does not present any flavour breaking, and in particular the disconnected part of eq. (2.4) vanishes. We remark that this is not the neutral pseudoscalar meson of tmQCD, but it is an interesting quantity to study with precise data on its own, in view of a possible use of mixed actions (the OS action for the valence quarks and tmQCD for the sea quarks). In fig. 7 the scaling behaviour of the connected correlator (OS pseudoscalar), is compared with the pseudoscalar meson in tmQCD where also the disconnected part is included (in both computations method $\mathbf{A}$ is used for the determination of the critical mass). For all the technical details of the computation of the disconnected part I refer to [42, 44]. The results show an $\mathrm{O}\left(a^{2}\right)$ scaling for both the pseudoscalar masses, even if there are indications that the neutral pseudoscalar meson for tmQCD (with the inclusion of the disconnected correlator) has reduced cutoff effects, within the rather large statistical errors. It is possible to give a very rough estimate of the 

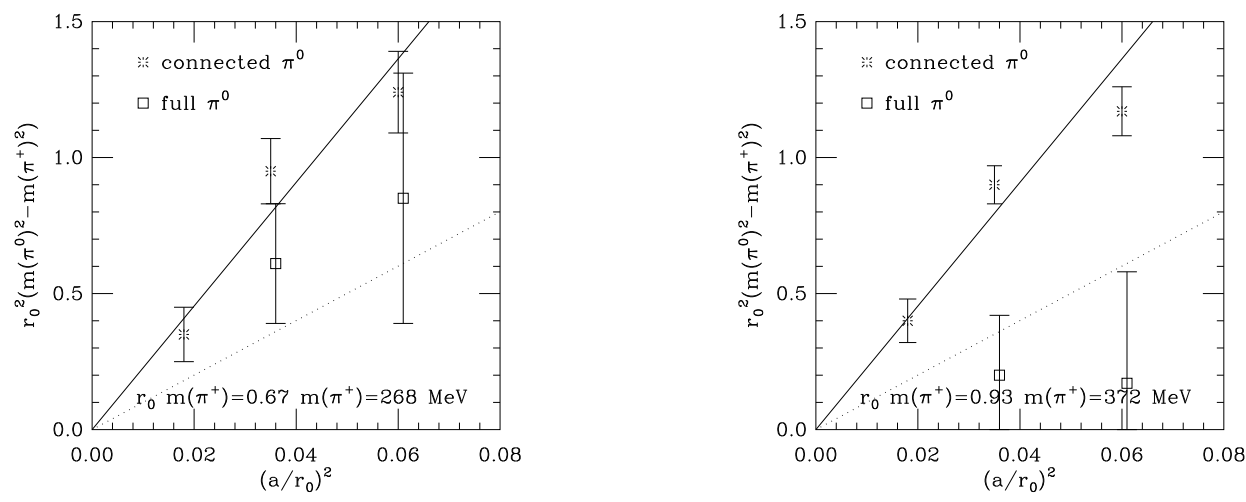

Figure 7: Scaling behaviour of the mass splittings between the neutral and the charged pseudoscalar masses for 2 values of $r_{0} m_{\mathrm{PS}}$. The open squared are the data for the neutral pseudoscalar meson with tmQCD, and the stars only the connected contribution (pseudoscalar meson with OS action). The full and dotted lines are an estimate of the $a^{2}$ dependence for the two pion splittings, making the hypothesis that $\mathrm{O}\left(a^{2}\right)$ effects are mass independent.

pion splitting $r_{0}^{2}\left(m_{\pi^{0}}^{2}-m_{\pi^{ \pm}}^{2}\right) \simeq c\left(a / r_{0}\right)^{2}$ with $c \simeq 10$ (with large errors). Comparing to a quenched simulation for naïve staggered fermions with Wilson gauge action [45], one finds a similar size of the flavour splitting encountered for the pion mass at a similar lattice spacing with a value $c \simeq 40$. For dynamical improved staggered fermions a value of $c \simeq 10$ has been found [46].

An interesting study of the flavour breaking effects was presented at this conference in [26]. To avoid the computation of disconnected diagrams in the quenched approximation a second doublet for strange and charm quarks is introduced following the strategy of [47]. Then the splitting on the kaon system is studied. In this study method $\mathbf{B}$ has been used for the determination of the critical mass. The results shown in fig. 8, indicates that, as expected, the flavour breaking effects vanish linearly with a rate of $a^{2}$, but that indeed they could be significant at a lattice spacing $a>0.1 \mathrm{fm}$. Another way to study flavour breaking effects is to consider $\Delta$ correlators. This was exploited in [23, 48], and studied in $\chi \mathrm{PT}$ in [49]. Recent results [44] with $N_{\mathrm{f}}=2$ dynamical tmQCD fermions and DBW2 gauge action (see next sections for details on the simulation parameters), indicate that at a lattice spacing of $a \simeq 0.12 \mathrm{fm}$ and a mass $\mu \simeq 12 \mathrm{MeV}$, the pion mass splitting even if with large errors, is consistent with zero.

3. $N_{\mathrm{f}}=2$

\subsection{Algorithmic improvements}

In the Lattice 2001 conference A. Ukawa presented [50] a rather impressing analysis on the possibility of simulating light quark masses with Wilson fermions. This was summarized with the now well known Berlin wall figure (see [51] for a recent update). Recently new algorithms 


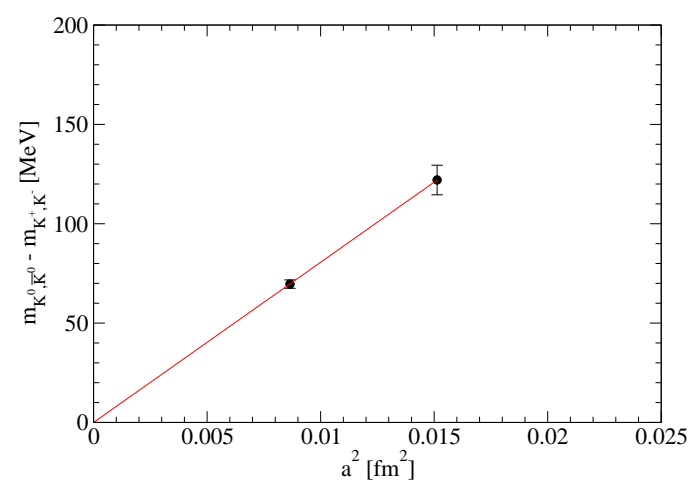

Figure 8: Scaling behaviour of the mass splitting between neutral and charged kaons.

[52. 53] have been proposed that have finally moved the wall to rather small quark masses. Both the algorithms are based on the standard HMC but have used new preconditioner. In [52] it was shown that with a domain decomposition (DD) preconditioning combined with a multiple time (mt) scale integrator [54], light quark masses $\left(m_{\pi}=294 \mathrm{MeV}\right)$ are reachable with Wilson fermions with remarkable performances. In [53] another very efficient preconditioner for the HMC algorithm has been introduced and tested, based on a mass preconditioner [55] (also known as Hasenbusch (H) acceleration) with again a multiple time scale integrator. In table 1 is summarized a rough comparison between the two algorithms, using different lattice actions, based on the so called cost figure $v=10^{-3}(2 N+3) \tau_{\text {int }}(P)$ introduced in [52]. The conclusion is that the algorithms have comparable performance down to pion masses of the order of $m_{\pi} \simeq 300 \mathrm{MeV}$. In fig. 9 is plotted the update of the Berlin wall figure. On the left panel there is a comparison between the results of [53, 56] (squares and diamond) and the results of [57] (circles). The lines are functions proportional to $\left(m_{\mathrm{PS}} / m_{\mathrm{V}}\right)^{4}$ (dashed) and $\left(m_{\mathrm{PS}} / m_{\mathrm{V}}\right)^{6}$ (solid). On the right panel it is shown a comparison between the Ukawa's formula in [50] (solid line) and the extrapolation of the results in [53] using a $\left(m_{\mathrm{PS}} / m_{\mathrm{V}}\right)^{4}$ (dashed) and a $\left(m_{\mathrm{PS}} / m_{\mathrm{V}}\right)^{6}$ (dotted) dependence for the data. The arrow indicates the physical pion to rho meson mass ratio. In addition there are also data points from staggered simulations (see [51] and references therein). In particular this plot indicates that running for one year a 1 Tflop sustained performance machine allows to generate at the physical point with $a \simeq 0.08 \mathrm{fm}$ and a lattice of $24^{3} \times 40,1000$ independent trajectories.

\subsection{Phase diagram of Wilson fermions}

In [58] the first study of tmQCD with $N_{\mathrm{f}}=2$ dynamical fermions was performed. Starting

\begin{tabular}{|c|c|c|c|c|c|}
\hline \hline Action & Algorithms & $r_{0} / a$ & $m_{\pi}[\mathrm{MeV}]$ & $v$ & $\tau_{\text {int }}$ \\
\hline $\mathrm{W}+\mathrm{W}$ & $(\mathrm{mt})(\mathrm{DD}) \mathrm{HMC}[$ [52] & $6.40(15)$ & 294 & $0.74(18)$ & $21(5)$ \\
tlSym+Wtm & $(\mathrm{mt})(\mathrm{H}) \mathrm{HMC}[$ [53] & $5.20(25)$ & 280 & $0.49(34)$ & $21(14)$ \\
\hline \hline
\end{tabular}

Table 1: Comparison of the 2 algorithms discussed in the text for a similar physical situation. 

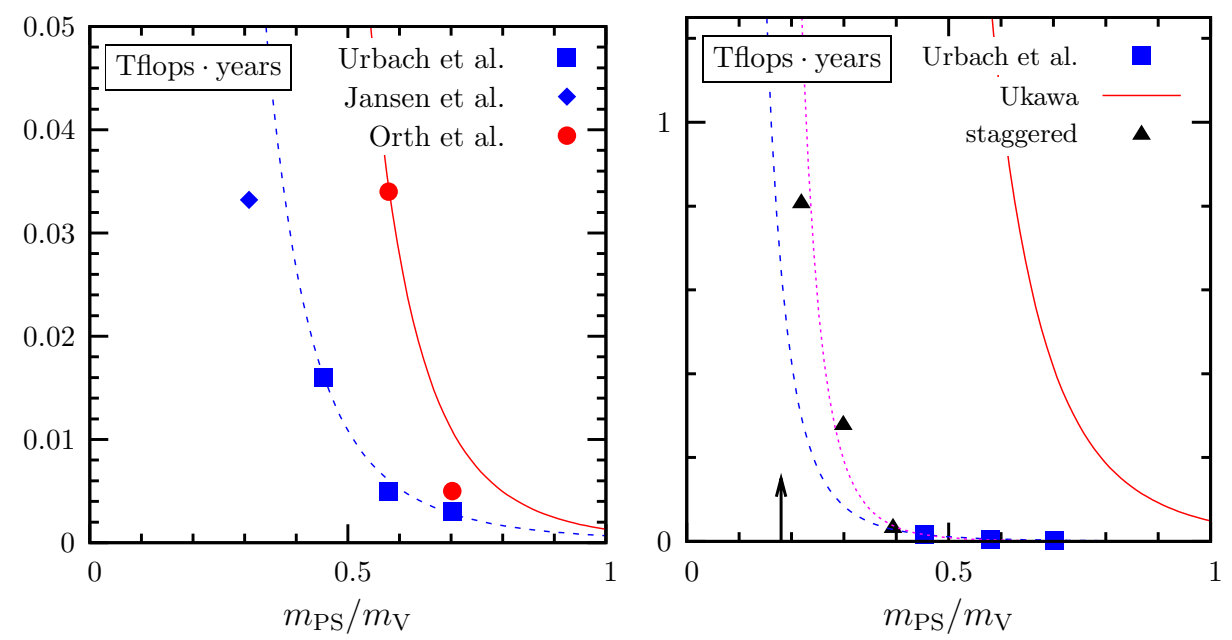

Figure 9: Computer resources needed to generate 1000 independent configurations of size $24^{3} \times 40$ at a lattice spacing of about $0.08 \mathrm{fm}$ in units of Tflops years as a function of $m_{\mathrm{PS}} / m_{\mathrm{V}}$. See text for a detailed description of the plots.

the exploration of a completely new territory, it is always good to remember a sentence of G. Parisi [59] "Let me describe a typical computer simulation: the first thing to do is to look for phase transitions". It is important to have then the correct understanding of the phase diagram with Wilson fermions in the 3 parameters space $\left(\beta=6 / g_{0}^{2}, m_{0}, \mu\right)$. To check that the results are not induced by the algorithm used it is always good to have at least 2 algorithms that reproduce the same results.

Indeed in [58] using the so called TSMB [60] and GHMC [55, 51] algorithms rather surprising results were found. The action used was Wilson gauge action combined with Wilson fermions with and without twisted mass. In particular at a lattice spacing of $a \approx 0.16 \mathrm{fm}$, strong evidence of a first order phase transition was found for a rather large range of values of twisted masses going from zero twisted mass to $\mu \simeq 100 \mathrm{MeV}$. This study reveals also that the phase transition tends to disappear increasing the value of $\mu$, it persists for $\mu=0$ and it is volume independent. A typical example of a MC history for the plaquette expectation value can be seen in fig. 10, where a cold and a hot start was performed. These results can help to see from a different point of view old numerical and theoretical works. In [62, 63] from a finite temperature study there was an indication of difficulties in observing a phase with spontaneous breaking of flavour and parity symmetry (Aoki phase) at $\beta>4.8$. In [64] the MILC collaboration found a surprising bulk first order phase transition for Wilson fermions at $\beta \simeq 4$.8. In [65] an analysis using the linear sigmamodel is performed, finding an indication of two possible patterns of symmetry breaking at finite lattice spacing. This observation was put on firmer theoretical basis in [18]. In this very important paper several interesting results and consideration were done, that, seen now from a different point a view, can help to understand the rather surprising numerical results obtained in [88]. In [18] for the first time the concept of chiral lagrangian at finite lattice spacing is given. The key point of the construction is the observation that the Pauli term in the effective Symanzik lagrangian transforms under chiral rotation exactly as does the mass term. I would like to add that this is also the key point for the automatic $\mathrm{O}(a)$ improvement for tmQCD at maximal twist. Neglecting the derivative 

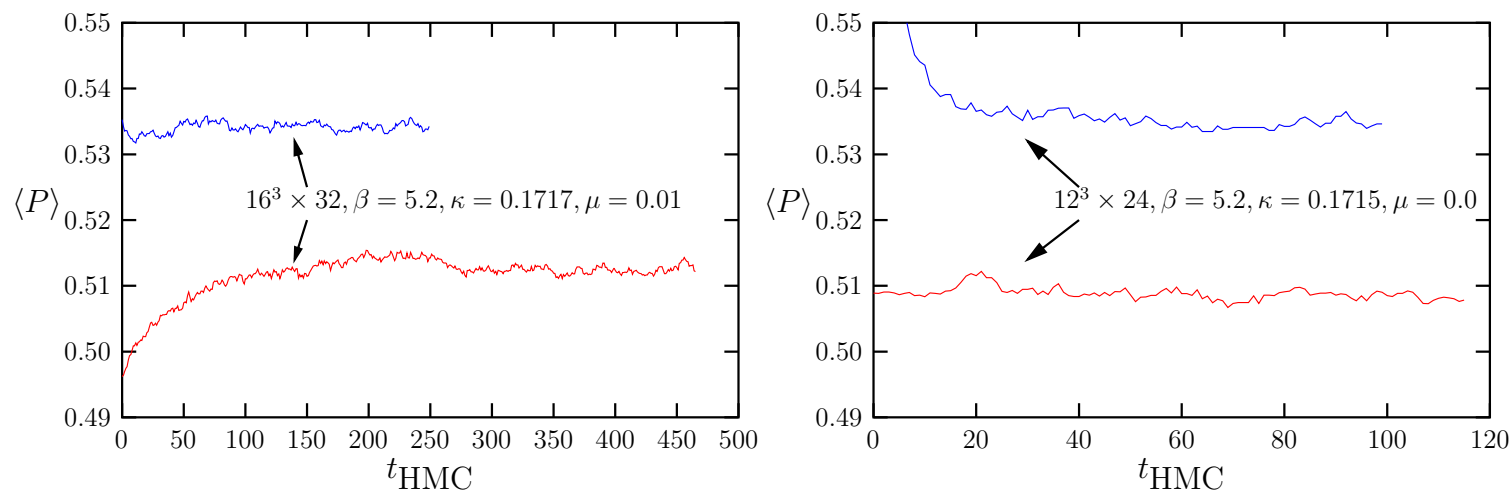

Figure 10: Metastable states at $\beta=5$.2. Left panel: $M C$ history of the average plaquette value with a twisted quark mass $\mu \simeq 10 \mathrm{MeV}$ and a lattice size $16^{3} \times 32$. Right panel: MC history of the average plaquette for pure Wilson fermions $(\mu=0)$ and a lattice size $12^{3} \times 24$.

interaction, being interested in the vacuum state, the potential of the effective chiral lagrangian reads

$$
\begin{gathered}
\mathscr{V}=-\frac{c_{1}}{4}\left\langle\Sigma+\Sigma^{\dagger}\right\rangle+\frac{c_{2}}{16}\left\langle\Sigma+\Sigma^{\dagger}\right\rangle^{2} \\
c_{1} \sim m^{\prime} \Lambda^{3} \quad c_{2} \sim m^{\prime 2} \Lambda^{2}+m^{\prime} a \Lambda^{4}+a^{2} \Lambda^{6} \quad m^{\prime}=m-a \Lambda^{2}
\end{gathered}
$$

where $\Sigma$ is the matrix that collects the Goldstone boson fields of the theory. We remark here that $m^{\prime}$ is a redefinition of the untwisted quark mass that includes the $\mathrm{O}(a)$ coming from the clover term. Up to $\mathrm{O}\left(a^{2}\right) m^{\prime}$ is proportional to the PCAC quark mass. The two terms in the potential become comparable when $m^{\prime} \sim a^{2} \Lambda^{3}$. In this region of quark masses the competition of these two terms causes a non-trivial vacuum structure that gives the following 2 scenarios [18] for the phase diagram of Wilson fermions: 1) The Aoki phase [1]; 2) The existence of a $1^{\text {st }}$ order phase transition [18]. The extension to tmQCD of these results is done in [66, 67, 68]. The result is summarized in fig. 11 where the $\mathrm{x}$-axis is $m^{\prime} / a^{2}$ and the $\mathrm{y}$-axis is $\mu / a^{2}$. A non-zero value of the twisted mass washes out the Aoki phase introducing an explicit breaking of flavour and parity symmetry. As can be seen from fig. 11 (left panel) the Aoki phase lies on the untwisted axis. In the second scenario in fig. 11 (right panel) the first order phase transition line extends into the twisted direction to a distance of $\mu_{\mathrm{c}} \approx a^{2} \Lambda^{3}$. The transition ends with a second order phase transition point, where the neutral pion mass vanishes. Several comments are in order now. The occurrence of one of the two scenarios depends on the sign of the coefficient $c_{2}$ proportional to the $\mathrm{O}\left(a^{2}\right)$ term in the chiral lagrangian. This coefficient $c_{2}$ depends on the choice of the gauge action, on the presence in the lattice action of the clover term and on the bare gauge coupling. An analysis with Wilson fermions of the two dimensional Gross-Neveau model [69] indicates that indeed both the scenarios describe the phase structure of Wilson fermions depending on the value of the couplings of the model. The analysis shows that at strong coupling there is an Aoki phase while at weak coupling the first order phase transition line sets in. This analysis has been recently extended for the twisted mass case [70], indicating even more complicated structures, like a coexistence of the two scenarios at the same value of the coupling.

Our present understanding of the lattice QCD phase diagram can be summarized as following. For values of the lattice spacing much coarser than $a=0.15 \mathrm{fm}$ there is a second order phase 


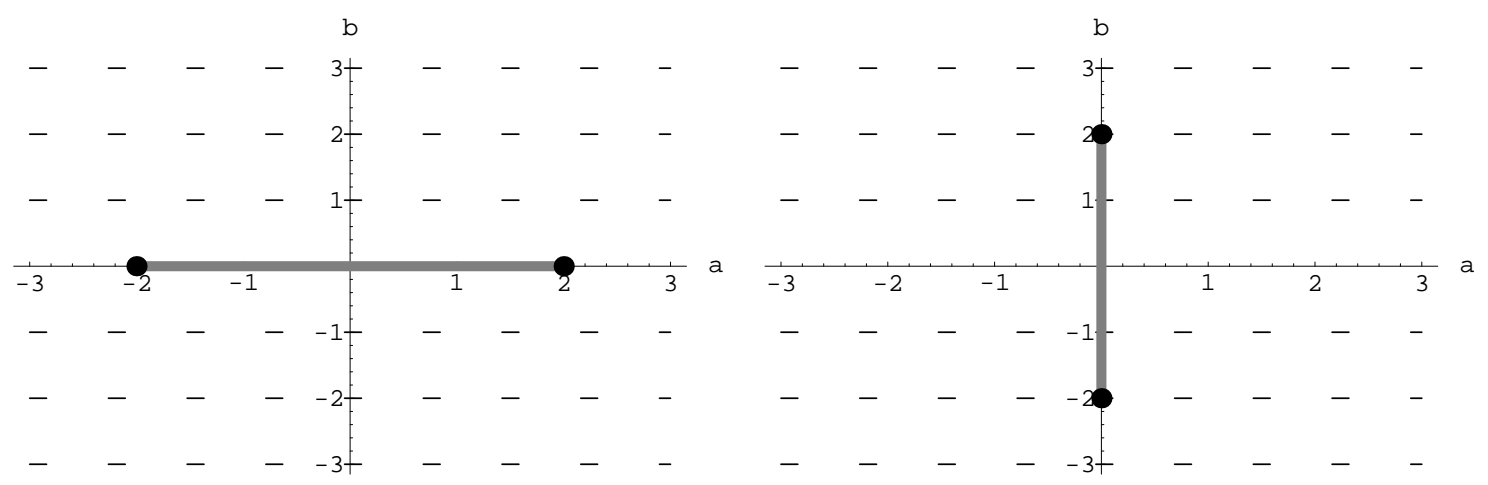

Figure 11: Left panel: the phase diagram of Wilson diagram according to $\chi \mathrm{PT}$ for $c_{2}>0$. Right panel: as the left panel but for $c_{2}<0$. The $\mathrm{x}$-axis is $m^{\prime} / a^{2}$ and the $\mathrm{y}$-axis is $\mu / a^{2}$.

transition from the standard lattice QCD phase to the Aoki phase [1, 71, 72]. For smaller values of the lattice spacing a first order phase transition appears [ [58, 73, 74, 75] that separates the positive quark mass from the negative quark mass phase. This first order phase transition is reminiscent of the continuum phase transition when the quark mass is changed from positive to negative values with the corresponding jump of the scalar condensate as the order parameter of spontaneous chiral symmetry breaking. The generic phase structure of lattice QCD is illustrated in fig. 12 and discussed in refs. [58, 73, 74].

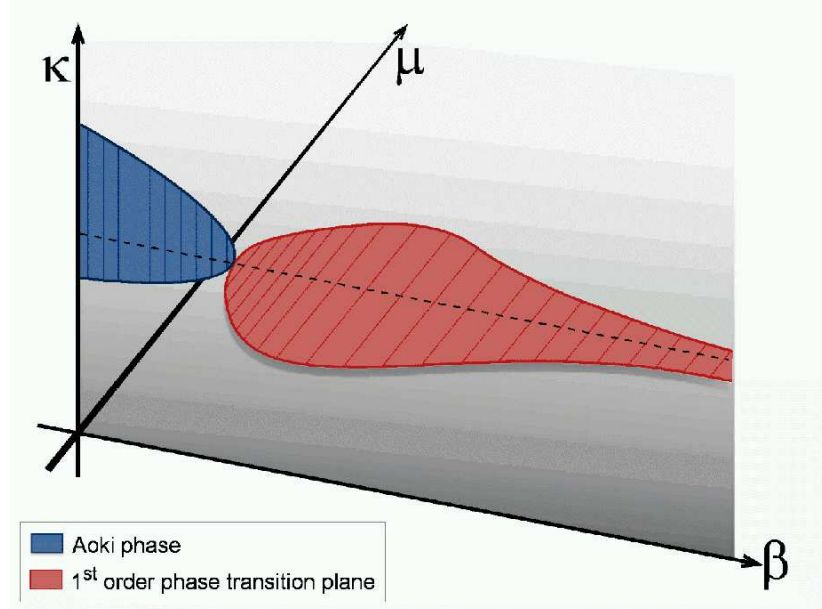

Figure 12: Current knowledge of the Wilson lattice QCD phase diagram as function of the inverse gauge coupling $\beta \propto 1 / g^{2}$, the hopping parameter $\kappa$ and the twisted mass parameter $\mu$.

\subsection{Minimal pion mass}

In the scenario with a first order phase transition the pseudoscalar mass $m_{\mathrm{PS}}$ cannot be made arbitrarily small both if the chiral point is reached from the untwisted or the twisted direction. Lowering the quark mass from the untwisted direction the algorithm will start to sample also in the region with negative masses. The minimal pion mass reachable will then depend on the algorithm 


\begin{tabular}{|l|l|l|}
\hline \multicolumn{1}{|c|}{$\beta=3.65$} & \multicolumn{1}{|c|}{$\beta=3.75$} & \multicolumn{1}{c|}{$\beta=3.90$} \\
\hline$a \mu=0.01$ & $a \mu=0.0094-0.005$ & $a \mu=0.0075-0.004$ \\
$a \approx 0.13 \mathrm{fm}$ & $a \approx 0.12 \mathrm{fm}$ & $a \approx 0.1 \mathrm{fm}$ \\
$L \approx 1.56 \mathrm{fm}$ & $L \approx 2 \mathrm{fm}$ & $L \approx 1.6 \mathrm{fm}$ \\
$\left(m_{\pi}\right)_{\min } \approx 450 \mathrm{MeV}$ & $\left(m_{\pi}\right)_{\min } \approx 400 \mathrm{MeV}$ & $\left(m_{\pi}\right)_{\min } \approx 280 \mathrm{MeV}$ \\
\hline
\end{tabular}

Table 2: Summary of the simulation parameters for dynamical runs of tmQCD with tlSym gauge action. The last line is an estimate of the minimal pion mass reachable without encountering metastabilities.

used and on the strength of the phase transition. Lowering the quark mass from the twisted direction there is a minimal pion mass given directly by the extension of the first order phase transition line, even if the twisted mass gives a sharp infrared cutoff in the sampling performed by the algorithm.

It therefore becomes important to understand the phase structure of lattice QCD as a prerequisite before starting large scale simulations. As we have seen the extension of the first order phase transition line in the twisted direction is proportional to the coefficient $\left|c_{2}\right|$. This coefficient depends both on the gauge action used and on the presence of the clover term in the lattice action. In [75] has been studied the lattice spacing dependence of of the first order phase transition with Wilson gauge action, taking as a measure of its strength, the gap between the two phases in the plaquette expectation value and in the PCAC quark mass. The qualitative estimate for the lattice spacing, where a minimal pion mass $m_{\pi} \simeq 300 \mathrm{MeV}$ could be reached, without being affected by the first order first transition is $0.07-0.1 \mathrm{fm}$.

It is suggestive that at the microscopic level the occurrence of this first order phase transition is accompanied by a massive rearrangement of the small eigenvalues of the Wilson-Dirac. This rearrangement could be suppressed by the use of a renormalization group improved or $\mathrm{O}\left(a^{2}\right)$ improved gauge actions, and indeed results from [76] indicate that metastabilities in the average plaquette observed for $N_{\mathrm{f}}=3$ dynamical Wilson fermions with a clover term (there is also an indication that the same metastabilities survive without a clover term for $N_{\mathrm{f}}=3$ ), can be suppressed replacing the Wilson gauge action with the Iwasaki action [77].

\subsection{Tree-level Symanzik improved gauge action}

The dependence of the phase diagram on the gauge action used and on the lattice spacing has been studied in a set of papers [ [58, 73, 74, 75] (see also [78] for a detailed summary of these results). The gauge actions so far studied can be parameterized by

$$
S_{\mathrm{G}}=\beta\left[b_{0} \sum_{x ; \mu<v}\left(1-\frac{1}{3} P^{1 \times 1}(x ; \mu, v)\right)+b_{1}\left(1-\frac{1}{3} P^{1 \times 2}(x ; \mu, v)\right)\right]
$$

with the normalization condition $b_{0}=1-8 b_{1}$. The parameters of the tree-level Symanzik action 779. $\left(b_{1}=-\frac{1}{12}\right)$ simulations are summarized in tab. 2. The last line indicates an estimate of the minimal pion mass reachable at the corresponding lattice spacings. In order to check for a possible phase transition and corresponding metastabilities a measure of the average plaquette value as a function of the hopping parameter $\kappa$ on runs that start from both a hot and a cold configuration has to be done. Since the metastability, if any, will show up around $\kappa_{c}$ (determined monitoring the 
PCAC mass $m_{\mathrm{PCAC}}$ at the corresponding fixed value of $\mu$ ) attention should be given to the hot and cold runs on $\kappa$-values closest to $\kappa_{c}$ only.

At $\beta=3.65, a \simeq 0.13 \mathrm{fm}, 12^{3} \times 24$ and $\mu \simeq 15 \mathrm{MeV}$ there are signs of a very nearby phase transition, as can be deduced from the steep rise in $\kappa$ of the plaquette expectation value (left panel in fig. 13, from a very slow thermalization and large fluctuations of the plaquette MC history value over several hundreds of trajectories (right panel in fig. 13). An estimate of the pseudoscalar mass, close to $\kappa_{\mathrm{c}}$, is $m_{\mathrm{PS}} \simeq 450 \mathrm{MeV}$.
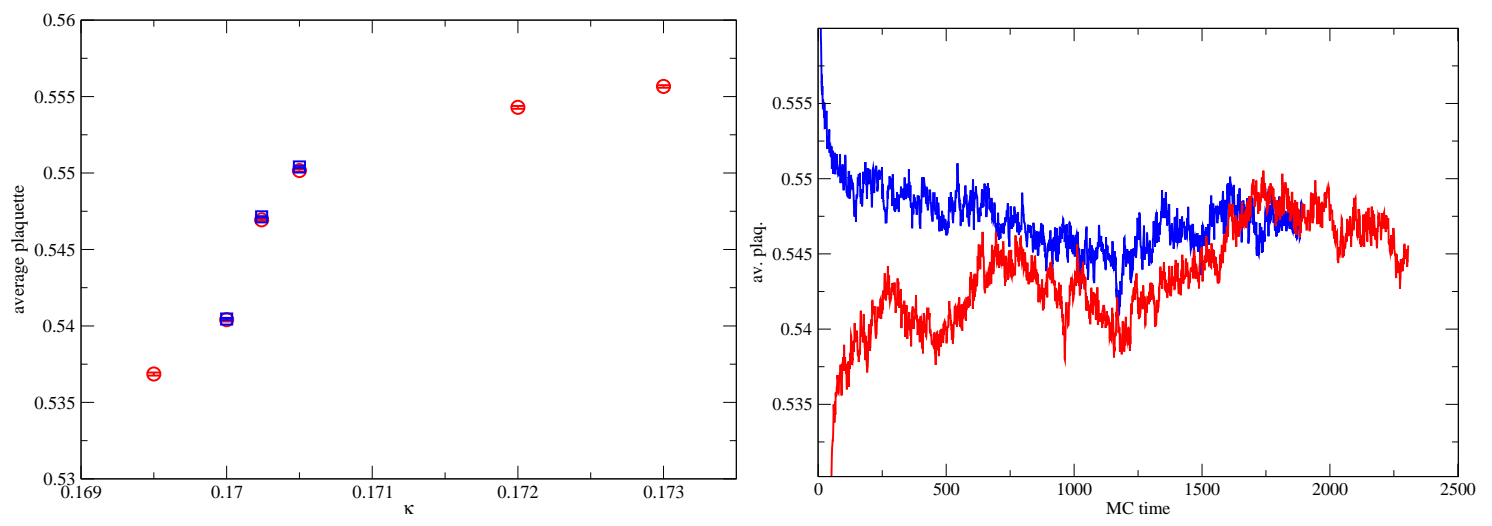

Figure 13: Left panel: Average plaquette value vs. $\kappa$ at $\beta=3.65, r_{0} \mu=0.038$ on a $12^{3} \times 24$ lattice from hot (red symbols) and cold starts (blue symbols). Right panel: Average plaquette MC time history for two runs at $\beta=3.65, r_{0} \mu=0.038, \kappa=0.17024$ on a $12^{3} \times 24$ lattice starting from hot (red line) and cold configuration (blue line).

At $\beta=3.75, a \simeq 0.12 \mathrm{fm}, 12^{3} \times 24$ and $\mu \simeq 8 \mathrm{MeV}$ there is a similar situation we have observed before at $\beta=3.65$ and $\mu \simeq 15 \mathrm{MeV}$. This is described by fig. 14 (left panel), where it is plotted the $\kappa$ dependence of the PCAC mass. This dependence is very useful to monitor a possible metastable critical point, since this shows up in a different extrapolated $\kappa_{\mathrm{c}}$, when the extrapolation is performed from positive or negative quark masses. A second twisted mass $\mu \simeq 15 \mathrm{MeV}$ has been simulated in a lattice $16^{3} \times 32$ around the critical point for this lattice spacing. Even if a strict check done with a hot and a cold start is not available at the moment the $\kappa$ dependence of the PCAC mass for this second value of $\mu$ suggests that the critical point is free from metastabilities. The pseudoscalar mass measured for the heaviest twisted mass is around $m_{\mathrm{PS}} \simeq 400 \mathrm{MeV}$. At $\beta=3.9, a \simeq 0.1 \mathrm{fm}, 16^{3} \times 32$ and $\mu \simeq 8$ and $15 \mathrm{MeV}$, there are no signs of metastabilities at the two corresponding critical points. In fig. 14 (right panel) is plotted the $\kappa$ dependence of the PCAC quark mass. The pseudoscalar masses obtained for the two values of $\mu$ are respectively $m_{\mathrm{PS}} \simeq 280$ and $450 \mathrm{MeV}$. We remark also that the physical volume at this $\beta$ value is rather small $L \simeq 1.6 \mathrm{fm}$, and results obtained for pure Wilson fermions [52, 57, indicate that for these quark masses and these volumes the finite size effects could be substantial. The estimate of the minimal pseudoscalar mass for this lattice spacing is then clearly only an upper bound.

\subsection{DBW2 gauge action}

In this section I summarize the results [73] obtained using the so called DBW2 gauge action [80] $\left(b_{1}=-1.4088\right)$. The parameters used in the simulations are summarized in tab. B. The 

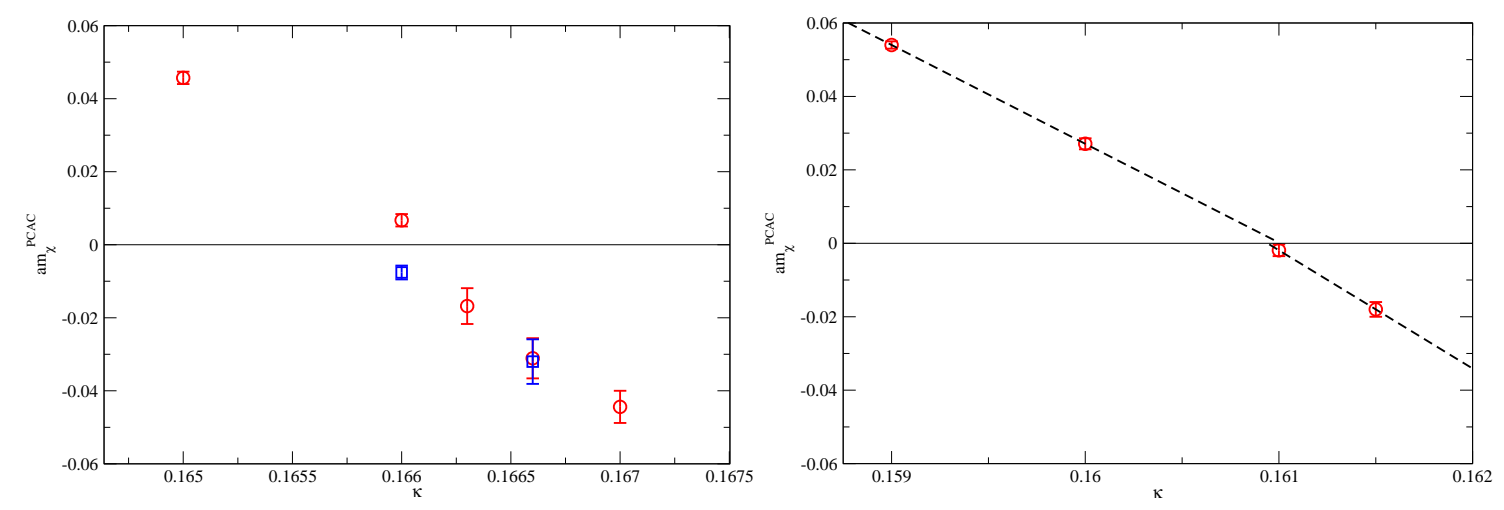

Figure 14: PCAC quark mass $m_{\mathrm{PCAC}}$ vs. $\kappa$ on a $16^{3} \times 32$ lattice. Left panel: $a \simeq 0.12 \mathrm{fm}, \mu \simeq 8 \mathrm{MeV}$. Right panel: $a \simeq 0.1 \mathrm{fm}, \mu \simeq 15 \mathrm{MeV}$.

\begin{tabular}{|l|l|}
\hline \multicolumn{1}{|c|}{$\beta=0.67$} & \multicolumn{1}{c|}{$\beta=0.74$} \\
\hline$a \mu=0.01$ & $a \mu=0.0075$ \\
$a \approx 0.19 \mathrm{fm}$ & $a \approx 0.12 \mathrm{fm}$ \\
$L \approx 2.3 \mathrm{fm}$ & $L \approx 2 \mathrm{fm}$ \\
$\left(m_{\pi}\right)_{\min } \approx 360 \mathrm{MeV}$ & $\left(m_{\pi}\right)_{\min } \approx 320 \mathrm{MeV}$ \\
\hline
\end{tabular}

Table 3: Summary of the simulation parameters for dynamical runs of tmQCD with DBW2 gauge action. The last line is an estimate of the minimal pion mass reachable without encountering metastabilities.

twisted mass for the two lattice spacing is kept roughly fixed to $\mu \approx 12 \mathrm{MeV}$. The last line indicates an estimate of the minimal pion mass reachable at the corresponding lattice spacings. Also for this gauge action several quantities have been computed. Here we concentrate as before on the PCAC mass and on the minimal pion mass. In contrast with the tlSym results here simulations at full twist were never performed, so the evidence for a metastability region can be deduced only indirectly from the dependence of the PCAC mass on the untwisted quark mass as discussed before. In fig. 15 is shown the $1 /(2 \kappa)$ dependence of the PCAC mass for the two lattice spacing used. At $a \approx 0.19$ $\mathrm{fm}$ there is an indirect evidence of a small metastability at full twist, that seems to disappear at $a \approx 0.12 \mathrm{fm}$. To summarize, there are first indications, that in order to reach pion masses of the order of $m_{\mathrm{PS}} \simeq 300 \mathrm{MeV}$ with tmQCD a gauge action like tlSym or DBW2 is appropriate. There are also first indications that this pion mass can be reached with DBW2 at slightly coarser lattices. In order to avoid possible large cutoff effects with DBW2 (see for example fig. 3 in [81]), or big coefficients in perturbative expansions, with the present data, the tlSym gauge action may be considered a better choice.

3.6 $N_{\mathrm{f}}=2+1+1$

The fact that tmQCD can be formulated only for an even number of flavours is not a limitation. Indeed using an off diagonal splitting, where the degenerate quark doublet has a different flavour orientation from the splitting between the quarks, in [82] it was shown that the determinant for non degenerate quarks is real and positive (see [47] for alternative formulations of tmQCD including 

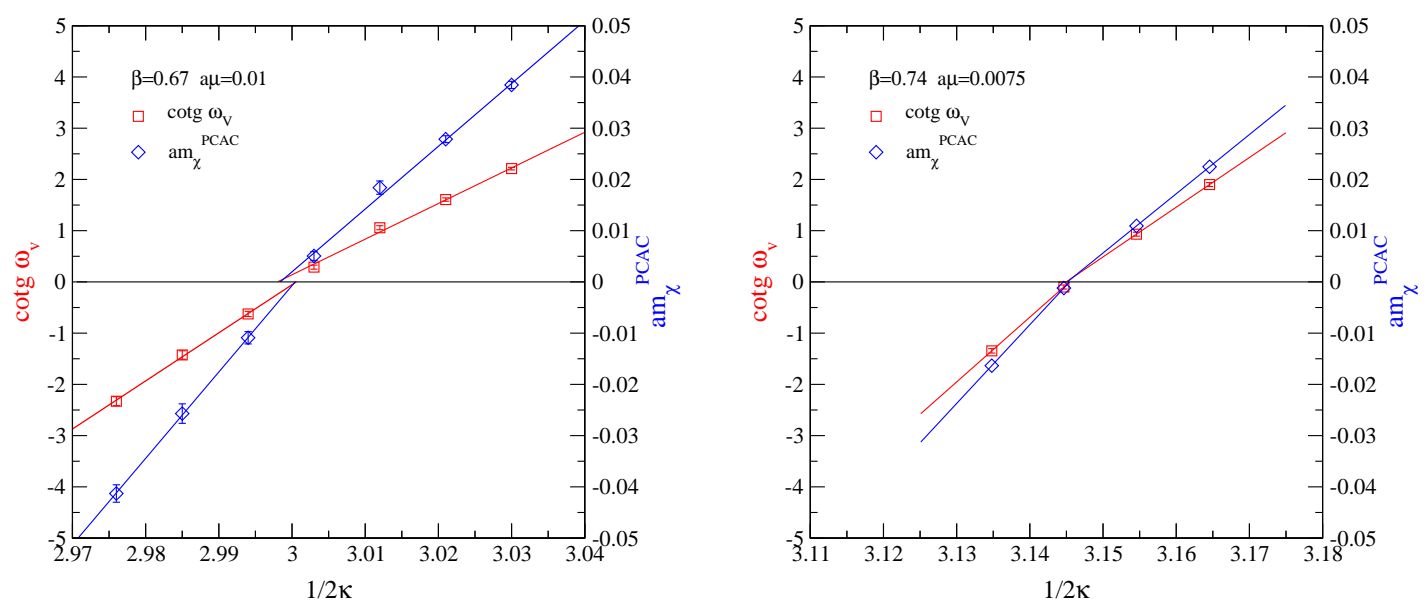

Figure 15: Determination of the critical hopping parameter $\kappa_{\mathrm{c}}$ by extrapolating to zero the untwisted PCAC quark mass $m_{\mathrm{PCAC}}$. The small discrepancy observed at $\beta=0.67$ (left panel) between extrapolations from positive and negative quark masses is probably a small effect of the first order phase transition. For $\beta=0.74$ (right panel), extrapolations from both sides give consistent results. An alternative way to fix the critical mass is also plotted (see [78] for details).

a non degenerate doublet). The only restriction of the construction in [82] is on the value of the ratio between renormalization constants of the pseudoscalar and scalar current. To give an example, fixing the values of the renormalized strange and charm quark masses, gives the following constraints

$$
\mu_{\mathrm{c}}^{\mathrm{R}} \simeq 1.5 \mathrm{GeV} \quad \mu_{\mathrm{s}}^{\mathrm{R}} \simeq 0.1 \mathrm{GeV} \Rightarrow \frac{Z_{\mathrm{P}}}{Z_{\mathrm{S}}}>0.875 .
$$

At this conference first results with dynamical $N_{\mathrm{f}}=2+1+1$ twisted quarks have been presented [78]. The simulations of the $N_{\mathrm{f}}=2+1+1$ theory are performed by a polynomial hybrid Monte Carlo algorithm (PHMC) [83]. The structure of the algorithm goes along the lines indicated in [84]. At this conference another variant of the PHMC to include the two non degenerate twisted quarks has been presented [85].

\section{Further results}

In this section I summarize further results concerning tmQCD. In [86] it has been presented a strategy to compute $B_{\mathrm{K}}$ and matrix elements related to the $\Delta I=1 / 2$ rule without mixing with operators with wrong chiralities, retaining all the properties of automatic $\mathrm{O}(a)$ improvement. The strategy is based on the usage of a mixed action (OS for valence quarks and tmQCD for sea quarks) [41]. In the quenched approximation $B_{\mathrm{K}}$ has been computed [87] in the continuum limit, using clover improved tmQCD and a non-perturbative renormalization without mixing (in the SF scheme). A strategy to compute $B_{\mathrm{B}}$ with tmQCD, along the lines of [88], has been proposed in [89], and along the lines of [41] in [90]. In [47] a strategy, based on clover improved tmQCD with $N_{\mathrm{f}}=4$, has been proposed to compute the renormalization of $K \rightarrow \pi$ matrix elements. In [21] the 
effect of a twisted mass term of the low-lying modes of the Wilson-Dirac operator and a remnant of the index theorem for twisted mass fermions has been discussed.

\section{Conclusions}

Several lessons come from quenched studies of tmQCD. With a particular and field theoretically well founded definition of the critical mass, automatic $\mathrm{O}(a)$ improvement is effective till small pion masses $\left(m_{\pi}=272 \mathrm{MeV}\right)$, and the residual $\mathrm{O}\left(a^{2}\right)$ cutoff effects are small. The bending phenomenon just results from big cutoff effects, that are reproducible with $\chi \mathrm{PT}$ at finite lattice spacing. The bending phenomenon is not present even at finite lattice spacing with a suitable choice of the critical mass. The flavour breaking is an issue and it has to be investigated with dynamical simulations. We have indications of the existence of an Aoki phase for quenched Wilson fermions at lattice spacings around $a \simeq 0.1 \mathrm{fm}$.

To perform dynamical simulations at small pion masses, algorithmic improvements are crucial, and now new algorithms allow to have efficient and performant simulations with Wilson fermions and most probably with staggered fermions.

We have a much better understanding of the phase structure of dynamical Wilson fermions. A theoretically well founded action (tlSym gauge and tmQCD fermion action) allows to perform dynamical simulations with $N_{\mathrm{f}}=2$ at pion masses smaller then $300 \mathrm{MeV}$ starting from a lattice spacing $a \simeq 0.1 \mathrm{fm}$, allowing matching with $\chi \mathrm{PT}$, and simulation with $N_{\mathrm{f}}=2+1+1$ flavours are just starting.

In many cases it has been shown that the renormalization properties of local operators related to very important phenomenological quantities, is continuum like.

Although presently not all aspects of tmQCD are fully investigated, tmQCD is an attractive and powerful discretization of lattice QCD, and it certainly belongs to the pool of well founded fermion actions that ought to be used to control the continuum limit of physical quantities of interest.

Acknowledgments: I thank the LOC for the stimulating atmosphere of the conference. I would like to thank F. Farchioni, I. Montvay, K. Nagai, M. Papinutto, E. Scholz, L. Scorzato, N. Ukita, C. Urbach, U. Wenger and I. Wetzorke for discussions and a most enjoyable collaboration, and in particular Karl Jansen for constant encouragement, and for a careful reading of this manuscript. I have profited from interesting and useful discussions with S. Aoki, O. Bär, R. Frezzotti, C. Michael, G. C. Rossi, S. Sharpe, S. Sint. This work is partially supported by the DFG Sonderforschungbereich/Transregio SFB/TR9-03.

\section{References}

[1] S. Aoki Phys. Rev. D30 (1984) 2653.

[2] ALPHA Collaboration, R. Frezzotti, P. A. Grassi, S. Sint and P. Weisz JHEP 08 (2001) 058 [hep-lat/0101001].

[3] ALPHA Collaboration, R. Frezzotti, S. Sint and P. Weisz JHEP 07 (2001) 048 hep-lat/0104014]. 
[4] ALPHA Collaboration, M. Della Morte, R. Frezzotti, J. Heitger and S. Sint JHEP 10 (2001) 041 [hep-lat/0108019].

[5] M. Della Morte, R. Frezzotti and J. Heitger Nucl. Phys. Proc. Suppl. 106 (2002) 260-262 hep-lat/0110166].

[6] R. Frezzotti and G. C. Rossi JHEP 08 (2004) 007 [hep-lat/ 0306014 ].

[7] R. Frezzotti, G. Martinelli, M. Papinutto and G. C. Rossi hep-lat/0503034.

[8] K. Symanzik, Some topics in quantum field theory, . Presented at 6th Int. Conf. on Mathematical Physics, Berlin, West Germany, Aug 11-21, 1981.

[9] K. Symanzik Nucl. Phys. B226 (1983) 187.

[10] K. Symanzik Nucl. Phys. B226 (1983) 205.

[11] B. Sheikholeslami and R. Wohlert Nucl. Phys. B259 (1985) 572.

[12] M. Lüscher, S. Sint, R. Sommer and P. Weisz Nucl. Phys. B478 (1996) 365-400 hep-lat/9605038].

[13] S. Aoki and O. Bär Phys. Rev. D70 (2004) 116011 hep-lat/0409006.

[14] XLF Collaboration, W. Bietenholz et. al. JHEP 12 (2004) 044 [hep-lat/0411001].

[15] S. R. Sharpe and J. M. S. Wu Phys. Rev. D71 (2005) 074501 hep-lat/0411021.

[16] S. R. Sharpe hep-lat/0509009.

[17] G. Munster and C. Schmidt Europhys. Lett. 66 (2004) 652-656 [hep-lat/0311032].

[18] S. R. Sharpe and J. Singleton, R. Phys. Rev. D58 (1998) 074501 hep-lat/9804028.

[19] G. Rupak and N. Shoresh Phys. Rev. D66 (2002) 054503 hep-lat/0201019.

[20] XLF Collaboration, K. Jansen, M. Papinutto, A. Shindler, C. Urbach and I. Wetzorke Phys. Lett. B619 (2005) 184-191 hep-lat/0503031.

[21] XLF Collaboration, K. Jansen, M. Papinutto, A. Shindler, C. Urbach and I. Wetzorke hep-lat/0507010. To appear in JHEP.

[22] S. Aoki and O. Bär hep-lat/0509002.

[23] A. M. Abdel-Rehim, R. Lewis and R. M. Woloshyn Phys. Rev. D71 (2005) 094505 [hep-lat/0503007].

[24] XLF Collaboration, K. Jansen, A. Shindler, C. Urbach and I. Wetzorke Phys. Lett. B586 (2004) 432-438 [hep-lat/0312013.

[25] A. M. Abdel-Rehim and R. Lewis Phys. Rev. D71 (2005) 014503 hep-lat/0410047.

[26] A. M. Abdel-Rehim, R. Lewis and R. M. Woloshyn hep-lat/0509056.

[27] ALPHA Collaboration, J. Garden, J. Heitger, R. Sommer and H. Wittig Nucl. Phys. B571 (2000) 237-256 [hep-lat/9906013].

[28] R. Frezzotti and S. Sint Nucl. Phys. Proc. Suppl. 106 (2002) 814-816 [hep-lat/0110140].

[29] R. Sommer Nucl. Phys. B411 (1994) 839-854 [hep-lat/9310022].

[30] ALPHA Collaboration, M. Guagnelli, R. Sommer and H. Wittig Nucl. Phys. B535 (1998) 389-402 [hep-lat/9806005]. 
[31] Zeuthen-Rome (ZeRo) Collaboration, M. Guagnelli et. al. Eur. Phys. J. C40 (2005) 69-80 [hep-lat/0405027].

[32] S. Capitani et. al. hep-lat/0510023.

[33] Zeuthen-Rome / ZeRo Collaboration, M. Guagnelli et. al. Nucl. Phys. B664 (2003) 276-298 [hep-lat/0303012].

[34] L. Giusti, P. Hernandez, M. Laine, P. Weisz and H. Wittig JHEP 04 (2004) 013 [hep-lat/0402002].

[35] V. Lubicz. Talk presented at this lattice conference.

[36] S. Sint, The Schrödinger functional with chirally rotated boundary conditions, . Talk presented at this lattice conference.

[37] R. Frezzotti and G. Rossi hep-lat/0507030.

[38] Y. Taniguchi hep-lat/0412024.

[39] S. Sint Nucl. Phys. B421 (1994) 135-158 hep-lat/9312079.

[40] ALPHA Collaboration, M. Della Morte et. al. Nucl. Phys. B713 (2005) 378-406 [hep-lat/0411025].

[41] R. Frezzotti and G. C. Rossi JHEP 10 (2004) 070 [hep-lat/0407002].

[42] XLF Collaboration, K. Jansen et. al. hep-lat/0507032.

[43] K. Osterwalder and E. Seiler Ann. Phys. 110 (1978) 440.

[44] F. Farchioni et. al. hep-lat/0509036.

[45] N. Ishizuka, M. Fukugita, H. Mino, M. Okawa and A. Ukawa Nucl. Phys. B411 (1994) 875-902.

[46] C. Aubin et. al. Phys. Rev. D70 (2004) 094505 [hep-lat/ 0402030 .

[47] C. Pena, S. Sint and A. Vladikas JHEP 09 (2004) 069 hep-lat/ 0405028 .

[48] A. M. Abdel-Rehim, R. Lewis and R. M. Woloshyn hep-lat/0509098.

[49] A. Walker-Loud and J. M. S. Wu Phys. Rev. D72 (2005) 014506 [hep-lat/0504001].

[50] CP-PACS and JLQCD Collaboration, A. Ukawa Nucl. Phys. Proc. Suppl. 106 (2002) 195-196.

[51] K. Jansen Nucl. Phys. Proc. Suppl. 129 (2004) 3-16 [hep-lat/0311039.

[52] M. Lüscher hep-lat/0409106.

[53] C. Urbach, K. Jansen, A. Shindler and U. Wenger hep-lat/0506011.

[54] J. C. Sexton and D. H. Weingarten Nucl. Phys. B380 (1992) 665-678.

[55] M. Hasenbusch Phys. Lett. B519 (2001) 177-182 [hep-lat/0107019].

[56] K. Jansen, A. Shindler, C. Urbach and U. Wenger hep-lat/0510064.

[57] B. Orth, T. Lippert and K. Schilling hep-lat/0503016.

[58] F. Farchioni et. al. Eur. Phys. J. C39 (2005) 421-433 [hep-lat/0406039].

[59] G. Parisi, Principles of numerical simulations, . In *Parisi, G.: Field theory, disorder and simulations* 447- 478. In *Les Houches 1988, Proceedings, Fields, strings and critical phenomena* 529-562.

[60] I. Montvay Nucl. Phys. B466 (1996) 259-284 [hep-lat/9510042]. 
[61] M. Hasenbusch and K. Jansen Nucl. Phys. B659 (2003) 299-320 [hep-lat/0211042].

[62] S. Aoki, A. Ukawa and T. Umemura Phys. Rev. Lett. 76 (1996) 873-876 [hep-lat/9508008].

[63] S. Aoki, T. Kaneda, A. Ukawa and T. Umemura Nucl. Phys. Proc. Suppl. 53 (1997) 438-441 [hep-lat/9612010].

[64] T. Blum et. al. Phys. Rev. D50 (1994) 3377-3381 hep-lat/9404006.

[65] M. Creutz hep-lat/9608024.

[66] G. Munster JHEP 09 (2004) 035 [hep-lat/0407006.

[67] L. Scorzato Eur. Phys. J. C37 (2004) 445-455 [hep-lat/0407023].

[68] S. R. Sharpe and J. M. S. Wu Phys. Rev. D70 (2004) 094029 hep-lat/ 0407025.

[69] T. Izubuchi, J. Noaki and A. Ukawa Phys. Rev. D58 (1998) 114507 [hep-lat/9805019].

[70] K.-I. Nagai and K. Jansen hep-lat/0510076,

[71] E.-M. Ilgenfritz, W. Kerler, M. Müller-Preussker, A. Sternbeck and H. Stüben Phys. Rev. D69 (2004) 074511 hep-lat/0309057].

[72] A. Sternbeck, E.-M. Ilgenfritz, W. Kerler, M. Müller-Preussker and H. Stüben Nucl. Phys. Proc. Suppl. 129 (2004) 898-900 hep-lat/0309059].

[73] F. Farchioni et. al. Eur. Phys. J. C42 (2005) 73-87 [hep-lat/0410031].

[74] F. Farchioni et. al. Nucl. Phys. Proc. Suppl. 140 (2005) 240-245 hep-lat/ 0409098 .

[75] F. Farchioni et. al. hep-lat/0506025.

[76] JLQCD Collaboration, S. Aoki et. al. hep-lat/0409016,

[77] Y. Iwasaki Nucl. Phys. B258 (1985) 141-156.

[78] F. Farchioni et. al. hep-lat/0509131.

[79] P. Weisz Nucl. Phys. B212 (1983) 1.

[80] T. Takaishi Phys. Rev. D54 (1996) 1050-1053.

[81] S. Necco Nucl. Phys. B683 (2004) 137-167 [hep-lat/0309017].

[82] R. Frezzotti and G. C. Rossi hep-lat/0311008.

[83] R. Frezzotti and K. Jansen Phys. Lett. B402 (1997) 328-334 [hep-lat/9702016].

[84] I. Montvay and E. Scholz Phys. Lett. B623 (2005) 73-79 hep-lat/0506006.

[85] T. Chiarappa, R. Frezzotti and C. Urbach hep-lat/0509154.

[86] R. Frezzotti and G. Rossi hep-lat/0509155.

[87] ALPHA Collaboration, P. Dimopoulos, J. Heitger, C. Pena, S. Sint and A. Vladikas Nucl. Phys. Proc. Suppl. 140 (2005) 362-364 hep-lat/0409026].

[88] M. Guagnelli, J. Heitger, C. Pena, S. Sint and A. Vladikas Nucl. Phys. Proc. Suppl. 106 (2002) 320-322 hep-lat/0110097].

[89] ALPHA Collaboration, F. Palombi, M. Papinutto, C. Pena and H. Wittig hep-lat/0509008,

[90] M. Della Morte Nucl. Phys. Proc. Suppl. 140 (2005) 458-460 hep-lat/0409012].

[91] C. Gattringer and S. Solbrig Phys. Lett. B621 (2005) 195-200 hep-lat/0503004]. 